\title{
Imaging of Tumor Spheroids, Dual-Isotope SPECT, and Autoradiographic Analysis to Assess the Tumor Uptake and Distribution of Different Nanobodies
}

Irati Beltrán Hernández, ${ }^{1,2}$ Rene Rompen, ${ }^{3}$ Raffaella Rossin, ${ }^{4}$ Katerina T. Xenaki, ${ }^{1}$ Eugene A. Katrukha, ${ }^{1}$ Klaas Nicolay, ${ }^{3}$ Paul van Bergen en Henegouwen, ${ }^{1}$ Holger Grüll, ${ }^{3,4,5}$ Sabrina Oliveira (i) $^{1,2}$

${ }^{1}$ Division of Cell Biology, Department of Biology, Faculty of Science, Utrecht University, Utrecht, The Netherlands

${ }^{2}$ Pharmaceutics, Department of Pharmaceutical Sciences, Faculty of Science, Utrecht University, Utrecht, The Netherlands

${ }^{3}$ Department of Biomedical Engineering, Eindhoven University of Technology, Eindhoven, The Netherlands

${ }^{4}$ Department of Minimally Invasive Healthcare, Philips Research Laboratories, Eindhoven, The Netherlands

${ }^{5}$ Department of Diagnostic and Interventional Radiology, University of Cologne, Faculty of Medicine and University Hospital of Cologne, Cologne, Germany

\begin{abstract}
Purpose: Recent studies have shown rapid accumulation of nanobodies (NBs) in tumors and fast clearance of the unbound fraction, making NBs exceptional tracers for cancer imaging. In this study, we investigate the combination of in vitro imaging of tumor spheroids, in vivo dualisotope single-photon emission computed tomography (SPECT), and ex vivo autoradiographic analysis of tumors to efficiently, and with few mice, assess the tumor uptake and distribution of different NBs.

Procedures: The irrelevant NB R2 (16 kDa) and the EGFR-targeted NBs 7D12 (16 kDa) and 7D12-R2 (32 kDa) were investigated. Confocal microscopy was used to study the penetration of the NBs into A431 tumor spheroids over time, using the anti-EGFR monoclonal antibody (mAb) cetuximab $(150 \mathrm{kDa})$ as a reference. Dual-isotope $\left[{ }^{111}\right.$ In]DOTA-NB/[ $\left.{ }^{177} \mathrm{Lu}\right] \mathrm{DOTA}-\mathrm{NB}$ SPECT was used for longitudinal imaging of multiple tracers in the same animal bearing A431 tumor xenografts. Tumor sections were analyzed using autoradiography.

Results: No binding of the irrelevant NB was observed in spheroids, whereas for the specific tracers an increase in the spheroid's covered area was observed over time. The NB 7D12 saturated the spheroid earlier than the larger, 7D12-R2. Even slower penetration was observed
\end{abstract}

\footnotetext{
The original version of this article was corrected/revised to include the complete graphic legend of Figure 3.

Irati Beltrán Hernández and Rene Rompen have equal contributions.

Klaas Nicolay passed away on January 10, 2017.

Electronic supplementary material The online version of this article (https:// doi.org/10.1007/s11307-019-01320-x) contains supplementary material, which is available to authorized users.
} 
for the large mAb. In vivo, the tumor uptake of 7D12 was 19-fold higher than R2 after co-injection in the same animal, and 2.5-fold higher than 7D12-R2 when co-injected. 7D12-R2 was mainly localized at the rim of tumors, while 7D12 was found to be more evenly distributed.

Conclusions: This study demonstrates that the combination of imaging of tumor spheroids, dualisotope SPECT, and autoradiography of tumors is effective in comparing tumor uptake and distribution of different NBs. Results were in agreement with published data, highlighting the value of monomeric NBs for tumor imaging, and re-enforcing the value of these techniques to accurately assess the most optimal format for tumor imaging. This combination of techniques requires a lower number of animals to obtain significant data and can accelerate the design of novel tracers.

Key Words: Nanobodies, Spheroids, Dual-isotope SPECT, Tumor imaging

\section{Introduction}

The discovery and design of new molecular imaging tracers are of great importance for early detection and diagnosis of cancer. Therefore, a wide range of tumor specific tracers have been developed for the imaging of solid tumors. Besides the metabolic positron emission tomography (PET) tracer 2-deoxy-2-[ $\left.{ }^{18} \mathrm{~F}\right]$ fluoro-D-glucose, proteins that bind to different tumor antigens are commonly used in the clinic for cancer imaging, and can vary in size by at least two orders of magnitude $\left(10^{0}-10^{2} \mathrm{~nm}\right)[1,2]$. In particular, monoclonal antibodies (mAbs, $150 \mathrm{kDa}$ ) are the most frequently used in the clinic [3]. However, mAbs are not ideal for tumor imaging due to long time intervals between tracer administration and imaging, and limited and heterogeneous distribution of the mAb inside tumors [4]. Molecular size is one of the well-recognized factors affecting tumor uptake and distribution [5]. Thus, many efforts have been made to develop smaller tracers to allow more rapid imaging and to reduce the radioactive burden for patients in case of nuclear imaging. Among antibody fragments, nanobodies (NBs, $16 \mathrm{kDa}$ ) have emerged as an attractive alternative format for molecular imaging. NBs consist of the variable region of the heavy-chain antibodies found in camelids and cartilaginous fish [6]. Fast penetration and homogeneous distribution into tumors, as well as high-contrast imaging rapidly after NB injection, are some of the advantages of using NBs for imaging purposes [7, 8]. In fact, clinical evaluation of NBs for PET imaging has thus far been encouraging [9].

Developing a nuclear tracer for the clinic requires in vitro characterization of the tracer, followed by in vivo assessment of its biodistribution and tumor targeting properties. To aid and accelerate this process, efforts are being made in order to develop in vitro and in vivo models, as well as imaging techniques for the preclinical setting [10]. One of such in vitro models is spheroids three-dimensional (3D) cell cultures. In this model, cell to cell contact and interactions are maintained in all three dimensions in order to grow and resemble an in vivo tumor. In contrast to the commonly used 2D cultures, these spheroids simulate the in vivo tumor environment and maintain most of the characteristics present in tumors [11]. Advances in imaging techniques also aid the development of imaging tracers. Dual-isotope single-photon emission computed tomography (SPECT) is a non-invasive imaging strategy used in several medical fields, such as cardiology [12] and neurology [13]. This imaging technique allows distinction of radiolabeled compounds both in space and over time, making possible the simultaneous detection of multiple tracers in a single subject. Importantly, dualisotope indium-111/lutetium-177 SPECT has been already used as a tool to evaluate small peptide tracers in the preclinical setting [14], and its use is extended to the NB field in the present study.

In this study, we investigate the use of in vitro 3D tumor spheroid models in combination with in vivo dual-isotope SPECT imaging, and ex vivo autoradiographic analysis of tumors, to efficiently assess the tumor uptake and distribution of different NB formats, while using a reduced number of animals. First, we have compared the irrelevant NB R2 $(16 \mathrm{kDa})$ with the EGFR-targeted monomeric NB 7D12 $(16 \mathrm{kDa})$, to assess the effect of binding specificity on tumor uptake. Thereafter, we have compared the tumor uptake and distribution of 7D12 with the dimeric NB 7D12-R2 (32 kDa), which has similar binding affinity to EGFR but with twice the molecular weight. This study demonstrates that this combination of in vitro, in vivo, and ex vivo imaging techniques is effective in evaluating the tumor uptake and distribution of the different NB formats, further encouraging the use of the monomeric format as a tracer for imaging of solid tumors.

\section{Materials and Methods}

\section{Cell Culture and Spheroid Formation}

The A431 cell line (epidermoid carcinoma) was purchased from the American Type Culture Collection (ATCC) and cultured in high-glucose Dulbecco's Modified Eagle's Medium (Lonza) supplemented with $10 \%$ fetal calf serum (GE Healthcare), $100 \mu \mathrm{g} / \mathrm{ml}$ streptomycin, and $100 \mathrm{U} / \mathrm{ml}$ penicillin (Invitrogen). To grow spheroids, the wells of an 8well plate (Nunc Lab-Tek II Chambered Slide 8-wells plates; 
Thermo Scientific) were coated with a thin layer of Matrigel (Corning) and incubated at $37{ }^{\circ} \mathrm{C}$ for $20 \mathrm{~min}$ to allow polymerization. A suspension of 20,000 cells was added per well and incubated at $37{ }^{\circ} \mathrm{C}$ for 1 week. The medium was refreshed every other day.

\section{In Vivo Studies}

Six-to-nine weeks old Balb/C nude female mice were purchased from Charles Rivers. A431 cells $\left(2 \times 10^{6}\right.$ cell/100 $\mu 1$ sterile PBS $)$ were subcutaneously injected in the left shoulder of the mice. When tumors reached a size of $100-300 \mathrm{~mm}^{3}$, mice were used for imaging or biodistribution studies. The animal welfare committee of the Maastricht University (The Netherlands) approved all animal studies. Experiments were performed according to the U.S. National Institutes of Health principles of laboratory animal care and the Dutch national law "Wet op de Dierproeven" (Std 1985, 336).

\section{Proteins and Labeling}

NBs R2 and 7D12 have been previously described $[15,16]$. The dimeric NB 7D12-R2 consists of these two NBs connected by a linker of 10 amino acids, being 7D12 the only EGFR binding unit. All NBs were produced and purified as previously described [17]. Cetuximab was purchased from Merck.

For in vitro studies, proteins were conjugated to the fluorophore Alexa Fluor 647 NHS ester (Life Technologies), purified, and analyzed by SDS-PAGE as described in [16] for the photosensitizer IRDye700DX, except that the labeling reaction took place for $1.5 \mathrm{~h}$. The concentration of the final conjugate and degree of labeling were calculated by measuring absorbance at 280 and $689 \mathrm{~nm}$, as described in the manufacturer's manual.

For in vivo studies, NBs were conjugated to 5 eq p-SCNBn-DOTA (Macrocyclics) in DMSO. The reaction was carried out for $30 \mathrm{~min}$ at $37{ }^{\circ} \mathrm{C}$ at $\mathrm{pH}$ 9. PD-10 columns (5 kDa MWCO, GE Healthcare) were used to purify the DOTA-NB conjugates. The concentration of each conjugate in PBS was measured by UV at $280 \mathrm{~nm}$ (NanoDrop, Thermo Fisher) or with a BCA assay (Thermo Fisher). The DOTANB conjugates were analyzed by SDS-PAGE on $12.5 \%$ homogeneous gels under non-reducing conditions. The conjugates were radiolabeled with $\left[{ }^{111} \mathrm{In}\right] \mathrm{Cl}_{3}$ or $\left[{ }^{177} \mathrm{Lu}\right] \mathrm{Cl}_{3}$ (Mallinckrodt and Perkin Elmer, respectively), in $0.2 \mathrm{M}$ HEPES $\mathrm{pH}$ or $0.2 \mathrm{M}$ ammonium acetate $\mathrm{pH} 5.5$, respectively. The mixtures were incubated for $1 \mathrm{~h}$ at $37{ }^{\circ} \mathrm{C}$ followed by a DTPA challenge, to remove loosely bound radiometal, and size exclusion purification (Zeba desalting cartridges, $7 \mathrm{kDa}$ MWCO, Thermo Fisher). Labeling yields were determined by radio-TLC using a phosphor imager (FLA-7000, Fujifilm). Aliquots of the labeling mixtures were applied on TLC-SG strips (Varian Inc.) which were eluted with $200 \mathrm{mM}$ EDTA in saline. In these conditions, the radiolabeled NB remains at the origin while free In-111 or Lu-177 migrates with a $0.9 \mathrm{Rf}$. In order to adjust the specific activity, the radiolabeled NBs were mixed with the respective unlabeled NB. For in vivo studies, radiolabeled NBs were added with gentisic acid to a $5 \mathrm{mg} / \mathrm{ml}$ final concentration to protect the conjugates from radiolysis.

\section{Binding Affinity of Labeled Proteins}

The apparent binding affinity of the labeled tracers was assessed with A431 cells as previously described [16]. Briefly, cells in 96-well plates were incubated for $2 \mathrm{~h}$ at $4{ }^{\circ} \mathrm{C}$ with a concentration range of NBs in DMEM (without phenol red, and with $25 \mathrm{mM}$ Hepes and $1 \% \mathrm{BSA}, \mathrm{pH}$ 7.2). After washing off unbound NBs, plates were scanned with the Odyssey scanner at $700 \mathrm{~nm}$ in the case of the Alexa 647labeled proteins. For the radiolabeled tracers, the content of the wells was finally dissolved in $0.5 \%$ SDS, buffered and radioactivity measured in a $\gamma$-counter (Wizard 1480, Perkin Elmer) using a dual-isotope protocol with crosscontamination correction. Apparent affinity $\left(\mathrm{K}_{\mathrm{D}}\right)$ was calculated with GraphPad Prism 7.

\section{Fluorescence Confocal Microscopy}

Spheroids were incubated with $25 \mathrm{nM}$ of Alexa 647-labeled tracers for different time intervals at $37{ }^{\circ} \mathrm{C}$. After each incubation time, unbound conjugate was washed away and spheroids were fixed in $4 \%$ paraformaldehyde (Merck). Background fluorescence was quenched with $100 \mathrm{mM}$ glycine (Sigma-Aldrich) and spheroids were permeabilized using $0.5 \%$ Triton X-100 (Sigma-Aldrich). Staining of the nuclei was performed with DAPI (Roche). In some cases, Factin was stained with phalloidin-488 (Thermo Fisher). Finally, the slides were mounted with SlowFade Gold (Invitrogen). Imaging was performed using a confocal laser scanning microscope LSM700 (Carl Zeiss Microscopy $\mathrm{GmbH}$ ) with $\mathrm{a} \times 40$ oil objective (EC Plan-NeoFluar $\times 40$ / 1.3 Oil DIC). Pictures of spheroids were taken through the middle z-stack section.

Confocal images were analyzed with the Image $\mathrm{J}$ software. The plugin Radial Profile was used to obtain the profile of fluorescence intensity of Alexa 647 along the radius of the spheroid. This profile was adjusted to the radius of the spheroid since overall the radii varied only $\pm 12.5 \mu \mathrm{m}$. Data were normalized and depicted as the fluorescence intensity from the rim to the core of the spheroid using GraphPad Prism 7. Profile plots display the fluorescence intensity profile of one representative spheroid per time point. The area under the curve (AUC) was calculated from the fluorescence profiles and plotted against time. A minimum of eight spheroids from at least two independent experiments were analyzed per time point. 


\section{In Vivo SPECT/CT Imaging and Ex Vivo Biodis- tribution Studies}

Imaging studies were performed with groups of four A431 tumor-bearing mice injected with $\left[{ }^{111} \mathrm{In}\right] \mathrm{DOTA}-7 \mathrm{D} 12$ (ca. $10 \mathrm{MBq} / 100 \mu \mathrm{l}),\left[{ }^{111} \mathrm{In}\right] \mathrm{DOTA}-7 \mathrm{D} 12 /\left[{ }^{177} \mathrm{Lu}\right] \mathrm{DOTA}-7 \mathrm{D} 12$, $\left[{ }^{111} \mathrm{In}\right]$ DOTA-7D 12/[ $\left[{ }^{177} \mathrm{Lu}\right]$ DOTA-R2, or $\left[{ }^{111} \mathrm{In}\right]$ DOTA7D12/[ ${ }^{177}$ Lu]DOTA-7D12-R2 (ca. $10 \mathrm{MBq}$ In-111 and $40 \mathrm{MBq}$ Lu-177 combined in $100 \mu \mathrm{l})$. All mice received a similar dose of NB (ca. $0.16 \mu \mathrm{mol} / \mathrm{kg}$ total NB dose). For imaging studies, the animals were anesthetized with isoflurane and imaging started right after injection of labeled NBs. Imaging was performed with a small-animal SPECT/CT scanner (NanoSPECT, Bioscan) equipped with four detectors and converging nine-pinhole collimators $(1.4 \mathrm{~mm}$ pinhole diameter, $1 \mathrm{~mm}$ resolution). Windows of a relative peak width of $10 \%$ were positioned around 171 and $245 \mathrm{keV}$ for In-111, and $208 \mathrm{keV}$ for Lu-177. In total 180 projections were acquired over three rotations. Immediately after imaging ( $c a .3 \mathrm{~h}$ post injection), the animals were euthanized, tissues and organs of interest were collected, and radiation was quantified with a $\gamma$-counter. The collected samples included blood, tumor, heart, lung, liver, spleen, pancreas, kidneys, bladder, intestines, muscle, femur, and brain. Because of the upper detection limit of the $\gamma$-counter, only half of each tumor and one middle slice from the kidneys were quantified. The other half of the tumor was snap frozen and used for digital autoradiography.

One group of tumor-bearing mice was injected with a similar dose of $\left[{ }^{111} \mathrm{In}\right]$ DOTA-7D12/[ $\left.{ }^{177} \mathrm{Lu}\right]$ DOTA-7D12 (ca. $0.16 \mu \mathrm{mol} / \mathrm{kg}$ total NB) labeled at a lower molar activity (2.5 MBq for In-111 and $10 \mathrm{MBq}$ for Lu-177 combined in $100 \mu \mathrm{l}$, injected in awake mice) and was euthanized $1 \mathrm{~h}$ post-injection. The animals were dissected and organs and tissues of interest were harvested and measured in a $\gamma$ counter.

\section{SPECT/CT Image Reconstitution}

SPECT images were reconstructed using HiSpect software (SciVis) with an ordered subset expectation maximization (OSEM) iterative reconstruction algorithm. CT images were reconstructed using an exact cone beam FPB algorithm with a Shepp Logan filter (100\%). SPECT and CT images were fused in InVivoScope (BioScan). CT images were used to select regions of interest in muscle, liver, and bone. For tumor and kidney, regions were selected based on the $75 \%$ threshold value of the maximum intensity pixel values per each organ.

\section{Autoradiographic $\beta$-Imaging}

Frozen tumor sections of $10 \mu \mathrm{m}$ were mounted on microscopy slides, thawed, covered with scintillation foil, and imaged with a BetaIMAGER DFine (Biospace Lab).
Radioactive signal was separated based on decay-rates and scaling was adjusted to match the difference in $\beta$ particle emission of In-111 to Lu-177. Analysis and quantification of the images were performed with $\mathrm{M} 3 \mathrm{Vi}$ sion (Biospace Lab). At least two images were quantified per each tumor.

\section{Statistical Analysis}

In vitro data were analyzed with GraphPad Prism 7 using one-way ANOVA with Tukey post-hoc test to compare between groups. For the in vivo data, significance between tumor or kidney uptake of different groups was calculated with a 2 -tailed $t$ test or one-way ANOVA. A value of $p \leq$ 0.05 was considered significant. Statistical significance was depicted as $* p \leq 0.05, * * p \leq 0.01, * * * p \leq 0.001$, and $* * * * p \leq 0.0001$.

\section{Results}

\section{Alexa 647-Labeled Proteins}

The degree of labeling (DOL) for the anti-EGFR NBs 7D12 and 7D12-R2 was 0.55 . In the case of cetuximab and R2, the DOL was 1.44 and 0.3 respectively. The remaining free fluorophore in the protein conjugate was in all cases less than $10 \%$. All conjugates were first analyzed by SDSPAGE to verify the purity and molecular size, and their binding affinity was assessed on monolayer cell cultures (Suppl. Fig. 1 a and b, see Electronic Supplementary Material (ESM)). The $\mathrm{K}_{\mathrm{D}}$ of the EGFR-targeted tracers remained in the low nanomolar range and was comparable to the unconjugated counterparts [17, 18] (Suppl. Fig. 1 e, see ESM). Importantly, the binding affinity of 7D12 and 7D12R2 was similar, while R2 did not show a specific binding to A431 cells.

\section{Penetration and Distribution of Proteins into Tumor Spheroids}

A431 spheroids showed progressive growth over time and consisted of a compact mass of cells with cortical actin distribution (Fig. 1 a). After more than 10 days in culture, the integrity of the spheroids was compromised, yielding empty spaces of different sizes. Therefore, during our experiments, we used 7-days-old tumor spheroids with a diameter of 150-200 $\mu \mathrm{m}$.

The bound EGFR-targeted NBs were observed deeper into spheroids over time, as indicated by the clear fluorescent signal on the cell membrane (Fig. 1 b). In contrast, the irrelevant NB R2 could not be detected inside the spheroids at any time point. Fluorescence signal of 7D12 and 7D12-R2 was detected in the core of spheroids as early as $15 \mathrm{~min}$ after tracer addition, and this signal increased over time until reaching the same 

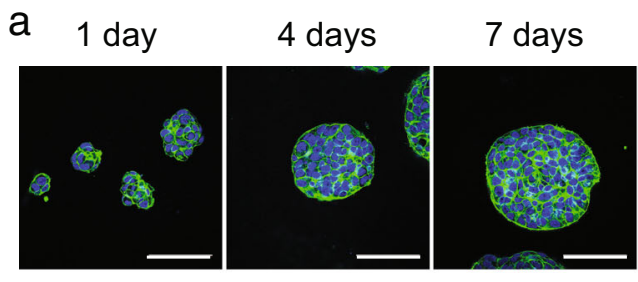

10 days
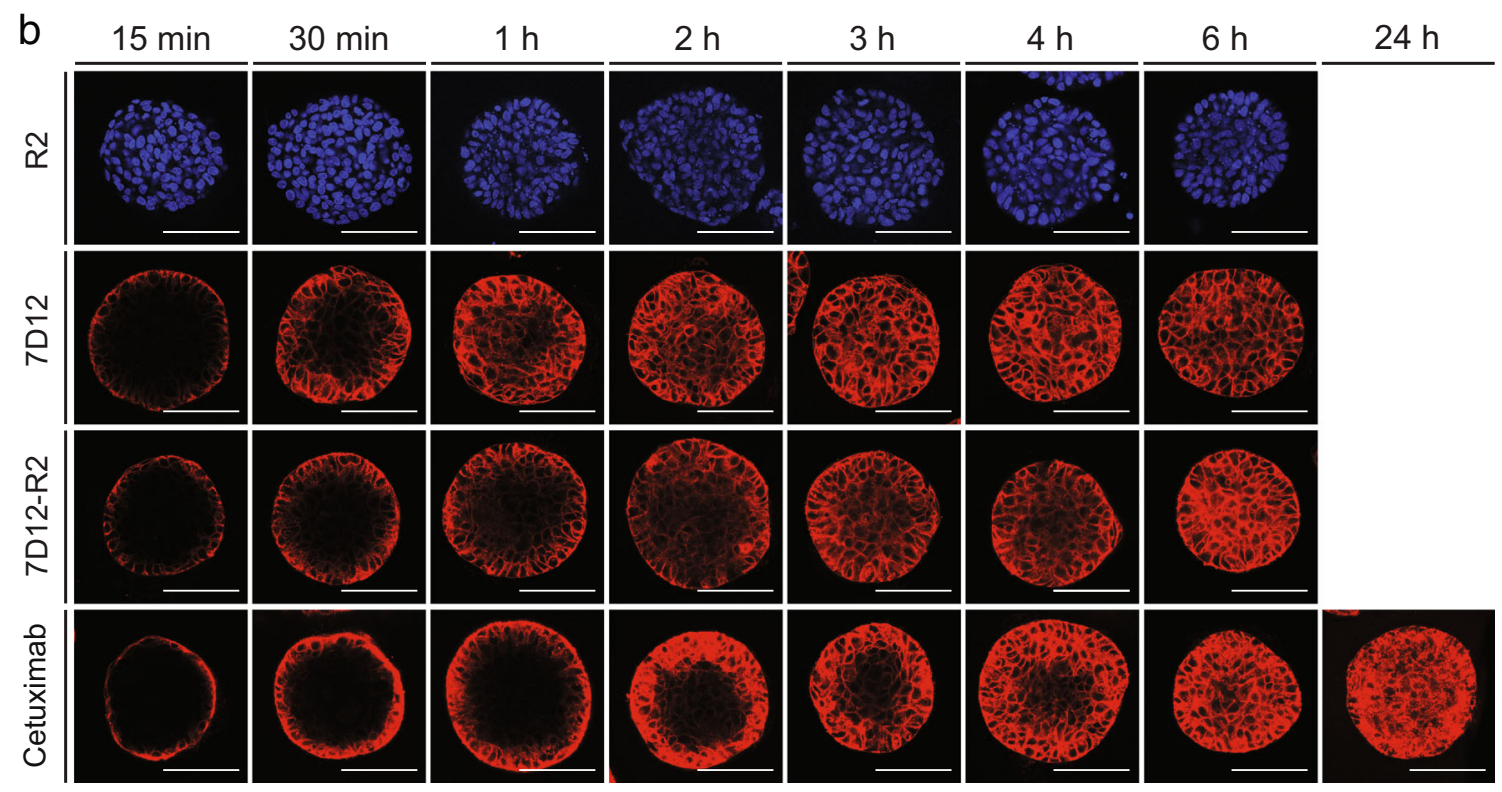

C

7D12

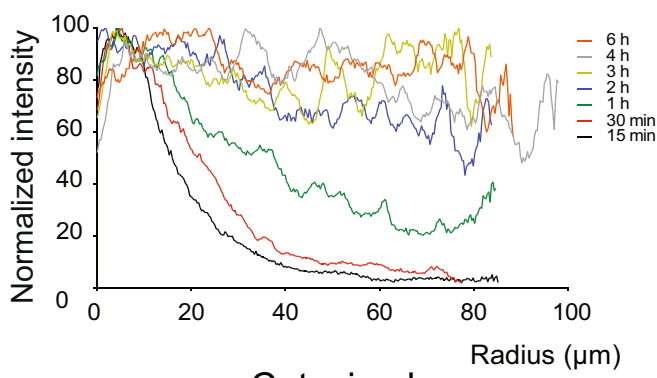

7D12-R2

\section{Cetuximab}
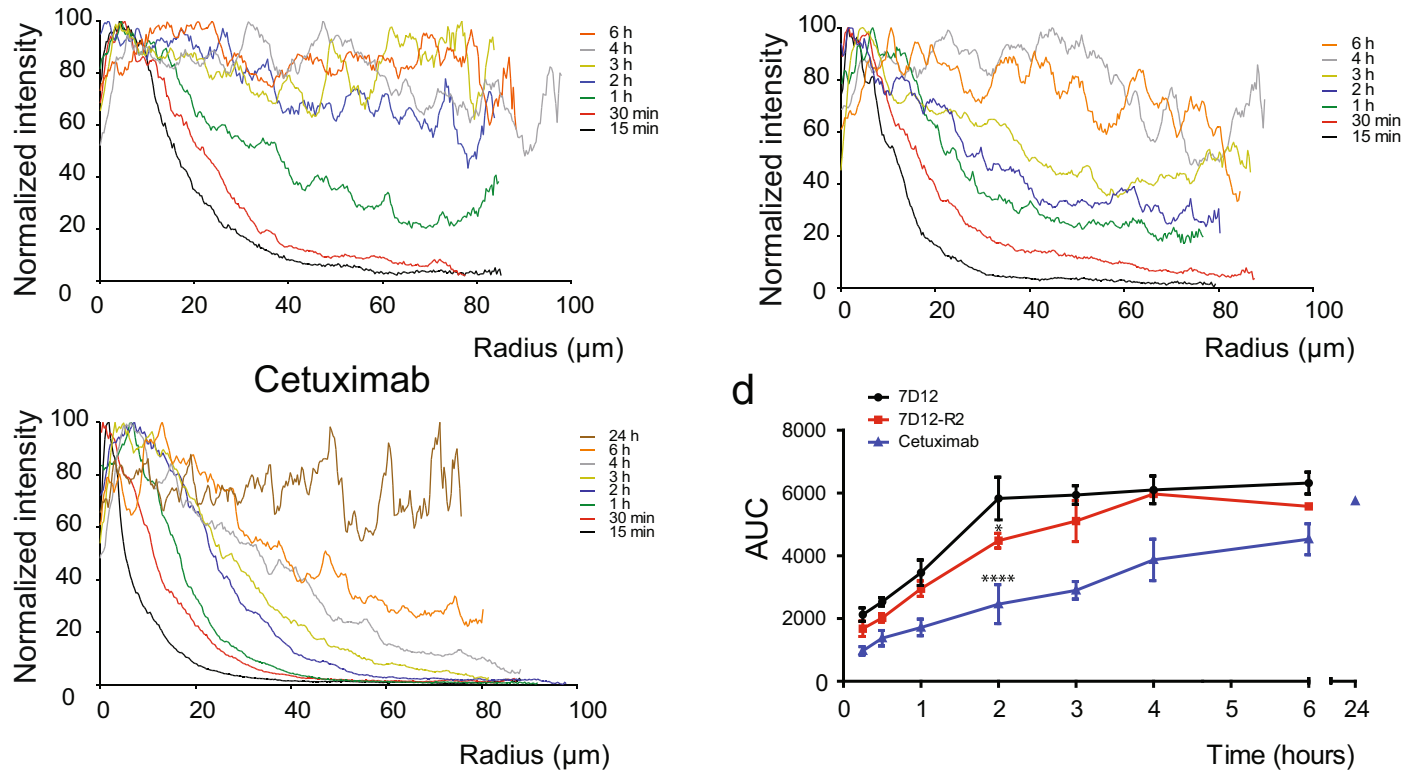

Fig. 1 Penetration and distribution of labeled proteins into tumor spheroids over time. a The growth of A431 spheroids was monitored over 10 days by taking confocal images at the middle section of spheroids. The spheroids were visualized by staining the nucleus of the cells with DAPI (blue) and the F-actin filaments with phalloidin-488 (green). b Confocal images of spheroids that were fixed after incubation with the Alexa 647-labeled proteins R2, 7D12, 7D12-R2, and cetuximab at defined time points (binding shown in red). In the case of R2, no Alexa 647 signal was detected bound to cells and, therefore, DAPI images of the same spheroids are shown instead, to depict the integrity of the spheroids. All images were linearly adjusted to the same level of contrast. Scale bar, $100 \mu \mathrm{m}$. $\mathbf{c}$ The fluorescence signal along the radius of spheroids was quantified from confocal images and displayed from the rim to the center of the spheroid. Graphs show one representative profile per time point and tracer. $\mathbf{d}$ The area under the curve of the fluorescence profiles was calculated and plotted against time as an estimation of the protein penetration into the spheroids in time. Statistical significance of the observed differences between each group and the 7D12 group was determined for the $2 \mathrm{~h}$ time point (mean $\pm \mathrm{SD}$ ). ${ }^{*} p \leq 0.05 ;{ }^{\star \star \star \star} p \leq 0.0001$. 
values as at the rim after 2 and $4 \mathrm{~h}$, respectively (Fig. $1 \mathrm{~b}$, c). The area under the curve (AUC) was determined from the fluorescence profiles of the tracers along the radius of the spheroids and plotted over time (Fig. 1 d). Some delay in tumor accumulation was observed for the dimeric 7D12-R2 tracer when compared with the monomeric 7D12 (Fig. 1c, d), while a more apparent delay was seen for cetuximab, which started to be observed in the core only after $3 \mathrm{~h}$ (Fig. $1 \mathrm{c}$ ). The delay of the mAb is more clearly visualized by the small slope of the spheroid coverage over time (Fig. 1 d).

\section{Radiolabeled Proteins}

The In-111 or Lu-177 labeling yield was comparable for all NBs, reaching more than $80 \%$ incorporation of In-111 or Lu-177 in all cases. After size exclusion purification, all tracers presented less than $5 \%$ free radiometal. Labeled NBs were analyzed on SDS-PAGE gel for their size and their apparent affinities were assessed by binding assays on monolayer cell cultures (Suppl Fig. 1 c, and d, see ESM). The apparent affinity of the NBs remained in the low nanomolar range after radiolabeling (Suppl. Fig. 1 e, see ESM). No specific binding to cells was observed for radiolabeled R2. The fact that molecular size and binding affinity remain comparable for each particular $\mathrm{NB}$, regardless of the fluorophore/isotope, suggests no detrimental effect of the different conjugations on binding properties, though direct comparisons between in vitro and in vivo data should be made carefully. Importantly, the main comparisons to be made are between the pairs of NBs that are subjected to the same labeling.

\section{Testing of Dual-Isotope SPECT and Comparison with Ex Vivo Biodistribution}

Tumor uptake of the NB 7D12 was clearly visible at 1 and $3 \mathrm{~h}$ post-injection in mice injected with the single-isotope $\left[{ }^{111}\right.$ In]DOTA-7D12 or with the combination of In-111 or Lu-177 radiolabeled 7D12, showing no differences along time (Fig. 2 a). Biodistribution of the tracers was determined both by SPECT imaging and by $\gamma$-counting of the respective organs (Fig. 2 b). Both techniques revealed a similar tumor uptake of the differently radiolabeled 7D12 over time, thus confirming that the distribution is not influenced by the radiolabel. A strong correlation was found for the uptake values in all examined organs measured by single and dual-isotope SPECT $\left(R^{2}=0.97\right.$ for In-111 and $R^{2}=0.97$ for Lu-177). Apart from the tumors, radioactivity uptake of both tracers was mainly observed in the kidneys (Fig. 2 b). Only there, differences were observed as the uptake for the dual-isotope group, detected by both SPECT imaging and $\gamma$-counting, was consistently higher than the single-isotope group. These differences may arise from the presence of crosstalk effects, particularly of Lu-177 in the acquisition window of In-111. Furthermore, metal chelates can have different renal clearance or uptake since these are also degraded in the kidney, and therefore different metal chelates can behave significantly different.

\section{Effect of Binding Specificity and of Tracer Molecular Size on Tumor Uptake and Biodistribution}

Tumor uptake was determined by both SPECT imaging and $\gamma$-counting after 1 and $3 \mathrm{~h}$ after tracer injection (Fig. $3 \mathrm{a}$, Suppl. Fig. 2 a, see ESM). The biodistribution of 7D12 was similar in all imaged animals for both time points. As calculated from SPECT imaging after 1 and $3 \mathrm{~h}, 7 \mathrm{D} 12$ presented a 19-fold higher tumor uptake than R2 after coinjection, whereas the examined organs showed a ratio close to 1 (Fig. 3 b, Suppl. Fig. 2 b, see ESM). When $\left[{ }^{111} \mathrm{In}\right]$ DOTA-7D12 and $\left[{ }^{177} \mathrm{Lu}\right]$ DOTA-7D12-R2 were coinjected, tumor uptake was 2.5 -fold higher for the monomeric NB 7D12, both after 1 and $3 \mathrm{~h}$, as measured by SPECT imaging. Importantly, SPECT imaging also showed a 2-3 times higher liver uptake of 7D12-R2 than 7D12. In addition, also the $\gamma$-counting results of blood and other organs (heart, pancreas, muscle, and lung) showed higher 7D12-R2 levels than 7D12 (Fig. 3 a). In general, $\gamma$-counting and SPECT data were strongly correlated (Supp. Fig. 3, see ESM), showing that a smaller tracer results in higher tumor uptake with lower background.

\section{Effect of Binding Specificity and of Tracer Molecular Size on Distribution Through Tumors}

Autoradiographic analysis of tumor sections indicated high accumulation of the In-111 and Lu-177 signal in the highly perfused rim of the tumors after injection of $\left[{ }^{111}\right.$ In]DOTA7 D $12 /\left[{ }^{177}\right.$ Lu $]$ DOTA-7D 12 or $\left[{ }^{111}\right.$ In]DOTA-7D $12 /$ $\left[{ }^{177} \mathrm{Lu}\right]$ DOTA-7D12-R2 (Fig. 4 a). Importantly, signal corresponding to 7D12 was more evenly distributed throughout the tumor. Quantification of autoradiographic images from all mice confirmed that the tumor uptake of 7D12 was similar in all animals, regardless of the coinjection with other NBs or the choice of the radionuclide (Fig. 4 b). Moreover, the presence of R2 in the tumor was negligible, whereas the signal from 7D12 was always higher than 7D12-R2.

\section{Discussion}

Many factors influence tumor uptake and distribution of tracers such as NBs, thus making it challenging to determine the most optimal tracer format for molecular imaging of solid tumors. In the present study, we have investigated the combination of imaging of in vitro tumor spheroids, in vivo dual-isotope SPECT, and ex vivo 


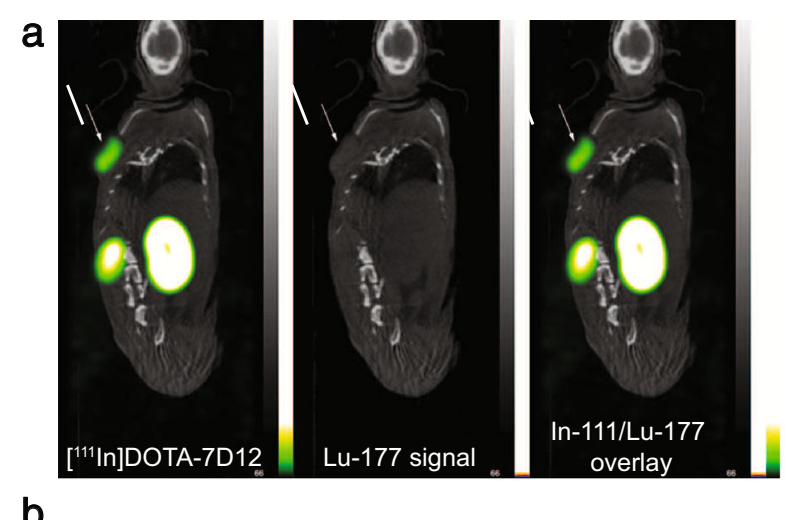

b
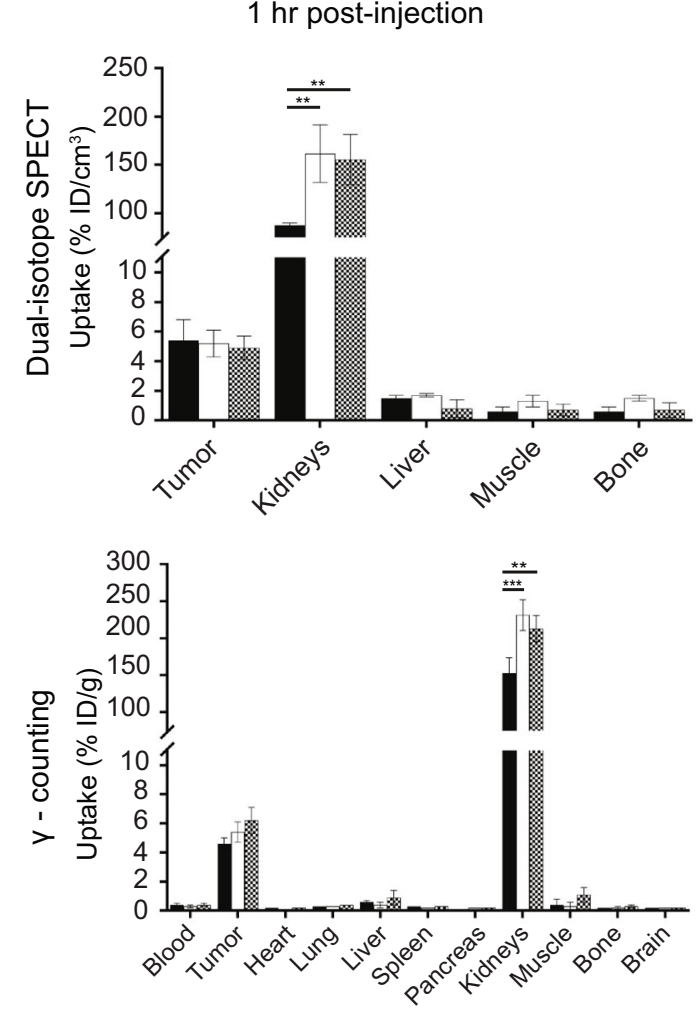
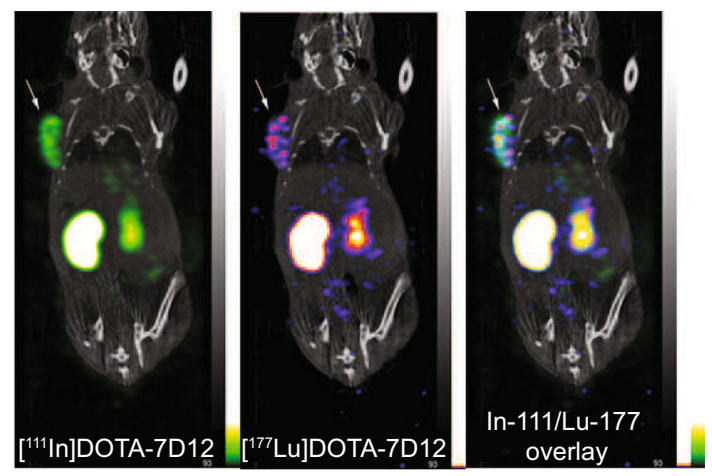

3 hrs post-injection
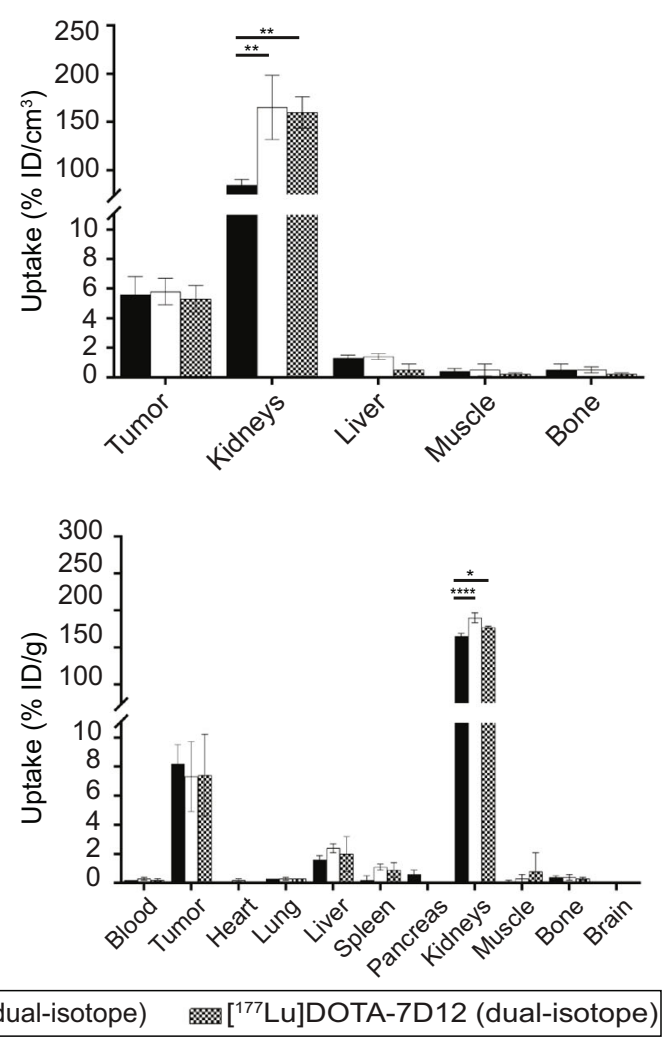

Fig. 2 Effect of radionuclide on imaging and biodistribution. a SPECT/CT coronal images of A431 tumor bearing mice $1 \mathrm{~h}$ after injection of [ ${ }^{111}$ In]DOTA-7D12 (left) and $3 \mathrm{~h}$ after injection of $\left.\left[{ }^{111} \mathrm{In}\right] \mathrm{DOTA}-7 \mathrm{D} 12 /{ }^{177} \mathrm{Lu}\right] \mathrm{DOTA}-7 \mathrm{D} 12$ (right). In-111 (In-111) signal and Lu-177 (Lu-177) signal were merged with CT images. Tumor location is indicated by arrows. b Biodistribution data obtained by single and dual-isotope SPECT imaging and $\mathrm{y}$-counting $1 \mathrm{~h}$ and $3 \mathrm{~h}$ after injection of ${ }^{111}$ In]DOTA-7D12 or $\left[{ }^{111}\right.$ In]DOTA-7D12/[ ${ }^{177}$ Lu]DOTA-7D12. Statistical significance of the observed differences between the kidney uptake of each dual-isotope group and the single-isotope group was determined (mean $\pm S D$ ). ${ }^{*} p \leq 0.05,{ }^{\star *} p \leq 0.01,{ }^{* \star *} p \leq 0.001$, and ${ }^{* \star \star *} p \leq$ 0.0001 .

autoradiographic analysis of tumor sections, to efficiently assess the tumor uptake and distribution of different NB formats, while using a reduced number of animals. Importantly, the three approaches yielded similar results. The similarity in the results obtained with the 3D spheroid model and the in vivo model suggests that the spheroids could predict the in vivo tumor distribution of the tracers. Although further studies with different targets and models are needed, 3D spheroids models have the potential to reduce the number of in vivo studies needed to compare different formats of tracers. Dual-isotope SPECT imaging was successfully confirmed here as a valuable technique for small animal NB imaging. Tumor uptake and biodistribution of each co-injected NB were quantified, with a strong linear relation with ex vivo $\gamma$-counting. The imaging and quantification of tracer couple within the same animal over time eliminates inter-animal differences, considerably reducing time and number of animals needed. Furthermore, 
a
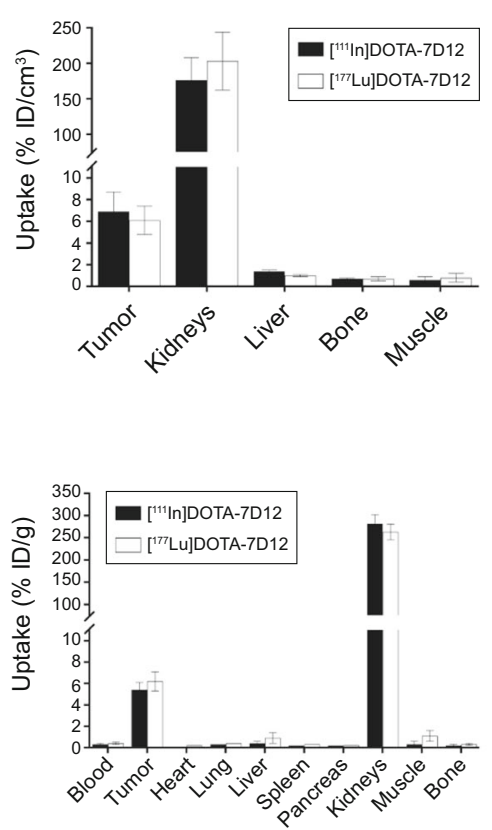

b
Dual-isotope SPECT
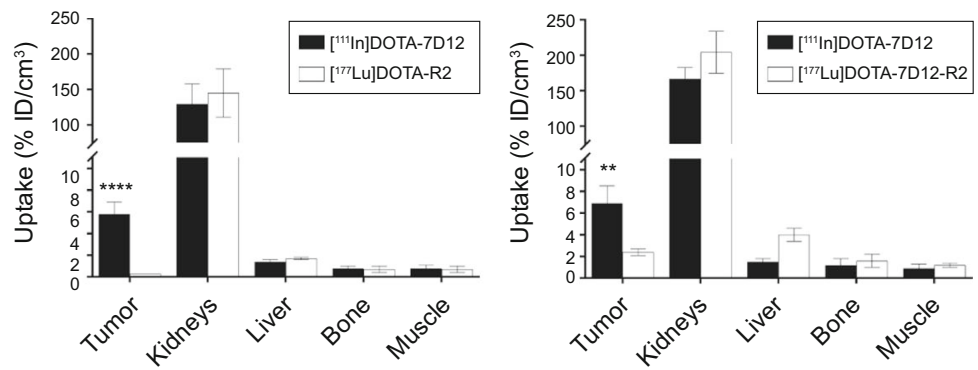

y-counting
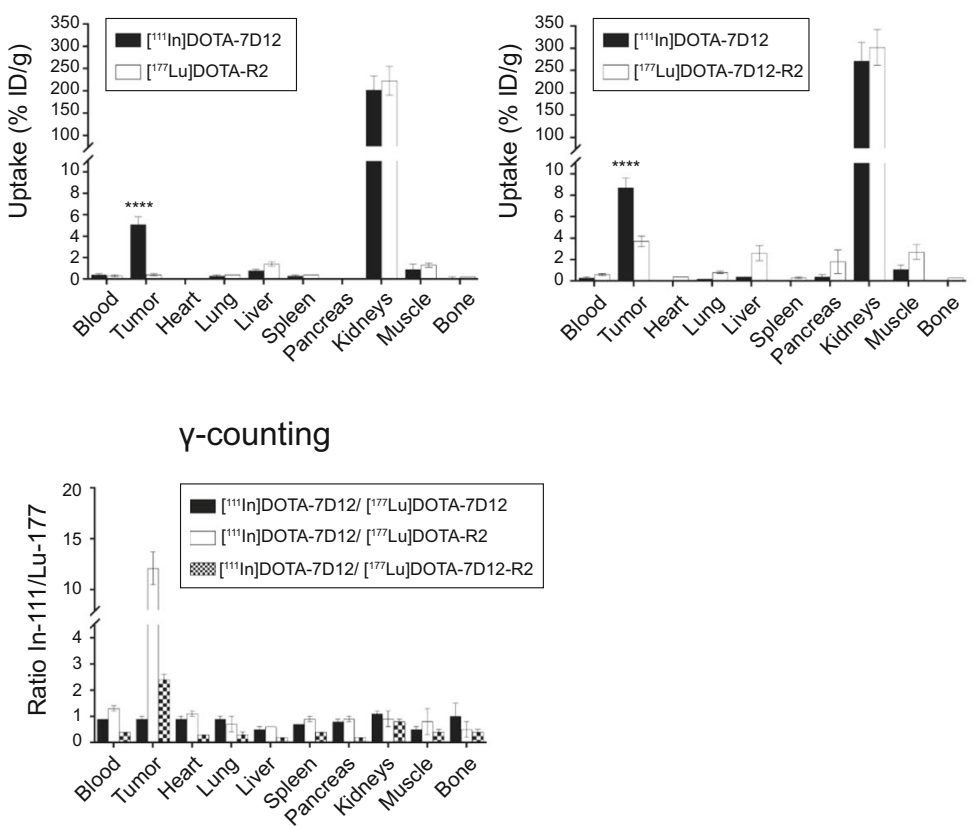

Fig. 3 Biodistribution of NB couples. a Biodistribution data from SPECT imaging and $y$-counting $1 \mathrm{~h}$ after injection of [ ${ }^{111}$ In]DOTA-7D12/[ $\left[{ }^{177}\right.$ Lu]DOTA-7D12, $\left[{ }^{111}\right.$ In]DOTA-7D12/[177Lu]DOTA-R2, or $\left[{ }^{111}\right.$ In]DOTA-7D12/ $\left[{ }^{177}\right.$ Lu]DOTA-7D12-R2 in tumor-bearing mice $(n=4)$. Statistical significance of the observed differences between the tumor uptake of each NB was determined (mean \pm SD). ${ }^{* \star} p \leq 0.01$ and ${ }^{* \star \star *} p \leq 0.0001$. b Ratios $\left[{ }^{111}\right.$ In]DOTA-NB/ $\left[{ }^{177}\right.$ Lu]DOTA-NB calculated from SPECT and $\mathrm{\gamma}$-counting biodistribution data (mean $\pm \mathrm{SD})$.

quantitative autoradiographic analysis of tumor sections allowed to more precisely compare the intratumoral distribution of the tracers.

In this study, we assessed the tumor accumulation of the irrelevant NB R2 and the EGFR-targeted NB 7D12. R2 was not retained in tumors in vivo, in agreement with the absence of accumulation into spheroids. On the contrary, 7D12 accumulated in spheroids and showed a 19-times higher tumor uptake than R2, when co-injected in mice. The considerably higher tumor uptake of a specific NB in comparison with an irrelevant $\mathrm{NB}$ is in agreement with previous studies evaluating single NBs with optical imaging [17] and single-isotope SPECT [19, 20]. We also assessed the behavior of tumor targeting NBs with different molecular size, but similar binding affinity (i.e., 7D12 and 7D12-R2). Our in vitro data showed that the monomeric NB 7D12 was able to more rapidly and more homogeneously distribute throughout spheroids over time, compared with the dimeric NB 7D12-R2. In addition, our in vitro data reinforces the notion that NBs penetrate tumors faster and deeper than mAbs, as documented in other studies [7, 17, 21]. Although tumor spheroids resemble many of the characteristics of an in vivo tumor, several aspects are not reproduced in this model, e.g., capillary extravasation and systemic clearance. Spheroids experiments showed that the accumulation of NBs into spheroids increased during the first 3-4 h, while the SPECT data showed no significant differences in tumor uptake of NBs between 1 and $3 \mathrm{~h}$ post-injection. This is probably caused by the rapid clearance of NBs via the kidneys (in vivo half-life $\sim 90 \mathrm{~min}$ [22]), which is not reproduced in the spheroid model. Importantly, 7D12 accumulated in tumors to a degree of $5-8 \% \mathrm{ID} / \mathrm{cm}^{3}$, which 
a

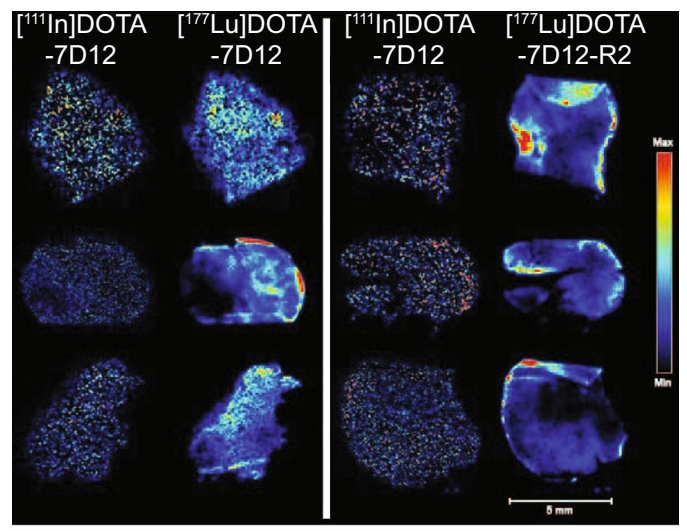

b

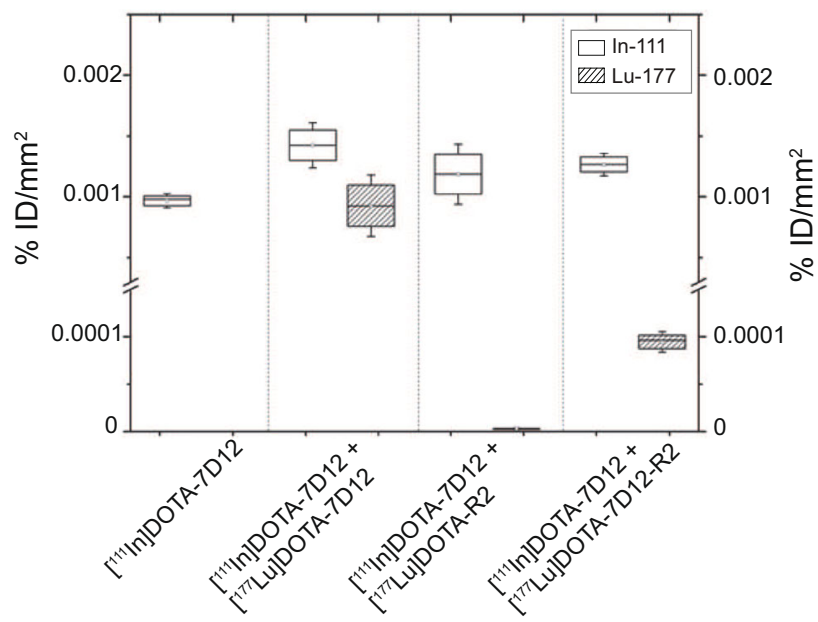

Fig. 4 Ex vivo autoradiography of tumor tissue and signal quantification. a Tumor-bearing mice were co-injected with $\left[{ }^{111}\right.$ In]DOTA-7D12/[77 Lu]DOTA-7D12 or $\left[{ }^{111}\right.$ In]DOTA-7D12/[77 Lu]DOTA-7D12-R2 and tumor sections were imaged based on decay rates. Representative tumor sections are shown. $\mathbf{b}$ Radioactivity quantification from autoradiographic images of tumor sections from mice injected with [ ${ }^{111}$ In]DOTA-7D12, $\left[{ }^{111}\right.$ In]DOTA-7D12/ $\left[{ }^{177}\right.$ Lu]DOTA-7D12, $\left[{ }^{111}\right.$ In]DOTA-7D12/ $\left[{ }^{177}\right.$ Lu]DOTA-R2, or $\left.\left[{ }^{111} \mathrm{In}\right] \mathrm{DOTA}-7 \mathrm{D} 12 /{ }^{177} \mathrm{Lu}\right] \mathrm{DOTA}-7 \mathrm{D} 12-\mathrm{R} 2$. Box plots display the range between first and third quartile, median and full data range (whiskers).

was similar to the reported $4.62 \% \mathrm{ID} / \mathrm{cm}^{3}$ for this NB using singe-isotope SPECT [18]. Remarkably, the increase of $16 \mathrm{kDa}$ for $7 \mathrm{D} 12-\mathrm{R} 2$ caused a 2.5 -times reduction in tumor uptake. This result underlines the effect of molecular size of the tracer on tumor uptake and is consistent with other studies evaluating similar NB formats with single-isotope SPECT $[19,20]$, thus supporting the reliability of the methods employed in the present study. Lastly, the autoradiographic images of tumor sections revealed that the dimeric NB had a limited penetration into the tumor in comparison to the monomer, which was also observed in vitro as a delayed penetration into spheroids.

Essential for imaging is the contrast between tumor and control tissues. In general, SPECT resulted in slightly lower uptake values in organs $(\sim 10 \%)$ than $\gamma$-counting, which is most likely due to ineffective crosstalk removal and partial volume effects at high activities [23]. This effect was more pronounced in the kidneys (approximately $30 \%$ of the injected dose was observed in each kidney), but values in kidneys were comparable with those from single-isotope imaging of EGFR-targeted NBs $[18,24]$. We also observed a retention of the dimeric 7D12-R2 in blood and other organs, which has been reported for other dimeric NBs as well [19].

Overall, the consistency of our results with published data confirms the value of the combination of techniques used here and demonstrates that this combination of techniques is useful to assess the tumor uptake and distribution of different (NB-based) tracers. Including imaging of $3 \mathrm{D}$ spheroids models in the in vitro characterization of new tracers, could lead to earlier identification of (un)promising candidates. The use of dual-isotope SPECT imaging in the subsequent preclinical studies could considerably reduce the number of animals needed. Lastly, autoradiographic analysis of the tumors could provide additional data on the distribution of tracers 
throughout tumors. In our case, the use of only four mice per group (per couple of NBs to investigate) led to unambiguous data because of the elimination of interanimal variations. In contrast, standard biodistributions studies would generally require six mice per tracer and per time point $[14,19]$.

\section{Conclusion}

A combination of in vitro experiments involving tumor spheroids and in vivo experiments with dual-isotope SPECT imaging and subsequent ex vivo autoradiography of tumors provided an accurate longitudinal study of different NB tracers, using a reduced number of animals. This combination of techniques can accelerate the design of novel targeting compounds, which could ultimately contribute to their progress to the clinic, for molecular imaging as well as other applications.

Funding. This study was funded by the Center for Translational Molecular Medicine-MAMMOTH project and by The Netherlands Organization for Health Research and Development, ZonMw — project 435000034.

\section{Compliance with Ethical Standards}

\section{Conflict of Interest}

All authors, except Dr. Rossin, declare that they have no conflict of interest. Dr. Rossin reports personal fees from Royal Philips Electronic and from Tagworks Pharmaceuticals.

\section{Ethical Approval}

All applicable institutional and/or national guidelines for the care and use of animals were followed.

Open Access This article is distributed under the terms of the Creative Commons Attribution 4.0 International License (http://creativecommons.org/licenses/by/4.0/), which permits unrestricted use, distribution, and reproduction in any medium, provided you give appropriate credit to the original author(s) and the source, provide a link to the Creative Commons license, and indicate if changes were made.

\section{References}

1. Khemthongcharoen N, Jolivot R, Rattanavarin S, Piyawattanametha W (2014) Advances in imaging probes and optical microendoscopic imaging techniques for early in vivo cancer assessment. Adv Drug Deliv Rev 74:53-74

2. Chakravarty R, Goel S, Cai W (2014) Nanobody: the "magic bullet" for molecular imaging? Theranostics 4:386-398

3. Mishani E, Hagooly A (2009) Strategies for molecular imaging of epidermal growth factor receptor tyrosine kinase in cancer. J Nucl Med 50:1199-1202

4. Jain RK (1990) Physiological barriers to delivery of monoclonal antibodies and other macromolecules in tumors. Cancer Res 50(Suppl):814s-819s

5. Xenaki KT, Oliveira S, van Bergen En Henegouwen PMP (2017) Antibody or antibody fragments: implications for molecular imaging and targeted therapy of solid tumors. Front Immunol 8:1287

6. Oliveira S, Heukers R, Sornkom J, Kok RJ, van Bergen en Henegouwen PMP (2013) Targeting tumors with nanobodies for cancer imaging and therapy. J Control Release 172:607-617
7. Kijanka M, Warnders F, El Khattabi M et al (2013) Rapid optical imaging of human breast tumour xenografts using anti-HER2 VHHs site-directly conjugated to IRDye $800 \mathrm{CW}$ for image-guided surgery. Eur J Nucl Med Mol Imaging 40:18-29

8. Xavier C, Blykers A, Vaneycken I, D'Huyvetter M, Heemskerk J, Lahoutte T, Devoogdt N, Caveliers V (2016) (18)F-nanobody for PET imaging of HER2 overexpressing tumors. Nucl Med Biol 43:247-252

9. Keyaerts M, Xavier C, Heemskerk J, Devoogdt N, Everaert H, Ackaert C, Vanhoeij M, Duhoux FP, Gevaert T, Simon P, Schallier D, Fontaine C, Vaneycken I, Vanhove C, de Greve J, Lamote J, Caveliers V, Lahoutte T (2016) Phase I study of 68Ga-HER2-nanobody for PET/CT assessment of HER2 expression in breast carcinoma. J Nucl Med 57:27-33

10. Wållberg H, Ståhl S (2013) Design and evaluation of radiolabeled tracers for tumor imaging. Biotechnol Appl Biochem 60:365-383

11. Antoni D, Burckel H, Josset E, Noel G (2015) Three-dimensional cell culture: a breakthrough in vivo. Int J Mol Sci 16:5517-5527

12. Blaire T, Bailliez A, Ben Bouallegue F et al (2017) Determination of the heart-to-mediastinum ratio of ${ }^{123} \mathrm{I}-\mathrm{MIBG}$ uptake using dualisotope $\left({ }^{123} \mathrm{I}-\mathrm{MIBG} /{ }^{99 \mathrm{~m}} \mathrm{Tc}\right.$-tetrofosmin) multi-pinhole CZT SPECT in patients with heart failure. J Nucl Med

13. Shiga T, Suzuki A, Sakurai K, Kurita T, Takeuchi W, Toyonaga T, Hirata K, Kobashi K, Katoh C, Kubo N, Tamaki N (2017) Dual isotope SPECT study with epilepsy patients using semiconductor SPECT system. Clin Nucl Med 42:663-668

14. Hijnen NM, de Vries A, Nicolay K, Grull H (2012) Dual-isotope ${ }^{1111} \mathrm{In} /{ }^{177} \mathrm{Lu}$ SPECT imaging as a tool in molecular imaging tracer design. Contrast Media Mol Imaging 7:214-222

15. Frenken LGJ, van der Linden RHJ, Hermans PWJJ, Bos JW, Ruuls RC, de Geus B, Verrips CT (2000) Isolation of antigen specific llama VHH antibody fragments and their high level secretion by Saccharomyces cerevisiae. J Biotechnol 78:11-21

16. van Driel PB, Boonstra MC, Slooter MD et al (2016) EGFR targeted nanobody-photosensitizer conjugates for photodynamic therapy in a pre-clinical model of head and neck cancer. J Control Release 229:93105

17. Oliveira S, van Dongen GA, Stigter-van Walsum M, Roovers RC, Stam JC, Mali W, van Diest P, van Bergen en Henegouwen P (2012) Rapid visualization of human tumor xenografts through optical imaging with a near-infrared fluorescent anti-epidermal growth factor receptor nanobody. Mol Imaging 11:33-46

18. Gainkam LO, Huang L, Caveliers V et al (2008) Comparison of the biodistribution and tumor targeting of two ${ }^{99 \mathrm{~m}} \mathrm{Tc}$-labeled anti-EGFR nanobodies in mice, using pinhole SPECT/micro-CT. J Nucl Med 49:788-795

19. Movahedi K, Schoonooghe S, Laoui D, Houbracken I, Waelput W, Breckpot K, Bouwens L, Lahoutte T, de Baetselier P, Raes G, Devoogdt N, van Ginderachter JA (2012) Nanobody-based targeting of the macrophage mannose receptor for effective in vivo imaging of tumor-associated macrophages. Cancer Res 72:4165-4177

20. Krasniqi A, Bialkowska M, Xavier C, van der Jeught K, Muyldermans S, Devoogdt N, D'Huyvetter M (2018) Pharmacokinetics of radiolabeled dimeric sdAbs constructs targeting human CD20. N Biotechnol 45:69-79

21. Bannas P, Lenz A, Kunick V, Well L, Fumey W, Rissiek B, Haag F, Schmid J, Schütze K, Eichhoff A, Trepel M, Adam G, Ittrich H, Koch-Nolte F (2015) Molecular imaging of tumors with nanobodies and antibodies: timing and dosage are crucial factors for improved in vivo detection. Contrast Media Mol Imaging 10:367-378

22. Roovers RC, Laeremans T, Huang L et al (2006) Efficient inhibition of EGFR signalling and of tumour growth by antagonistic anti-EGFR nanobodies. Cancer Immunol Immunother 56:303-317

23. Pretorius PH, King MA (2009) Diminishing the impact of the partial volume effect in cardiac SPECT perfusion imaging. Med Phys $36: 105-115$

24. Gainkam LO, Keyaerts M, Caveliers V et al (2011) Correlation between epidermal growth factor receptor-specific nanobody uptake and tumor burden: a tool for noninvasive monitoring of tumor response to therapy. Mol Imaging Biol 13:940-948

Publisher's Note Springer Nature remains neutral with regard to jurisdictional claims in published maps and institutional affiliations. 